ISSN: 0210-1696

DOI: http://dx.doi.org/10.14201/scero2015464724

\title{
MIEDOS, ESPERANZAS Y \\ REIVINDICACIONES \\ DE PADRES DE NIÑOS CON TEA
}

\section{Fears, Hopes and Demans by Parents of Children With ASD}

\author{
Lourdes Moro GutiérRez \\ Departamento de Psicología Social y Antropología. Facultad de Psicología. Universidad de \\ Salamanca. Avda. de la Merced, 109-131. 37005 Salamanca \\ moro@usal.es \\ Cristina Jenaro Río \\ INICO/Departamento de Personalidad, Evaluación y Tratamiento Psicológicos. Facultad de \\ Psicología. Universidad de Salamanca \\ María Solano SÁnchez \\ Máster en Antropología: Salud y Desarrollo Comunitario. Departamento de Psicología Social y \\ Antropología. Facultad de Psicología. Universidad de Salamanca
}

Recepción: 27 de agosto de 2015

Fecha de aceptación definitiva: 3 de noviembre de 2015

Biblid. [0210-1696 (2015) vol. 46 (4), n. ${ }^{\circ} 256$, octubre-diciembre; 7-24]

Resumen: El término "trastornos del espectro del autismo" (TEA) designa a un conjunto de condiciones muy variables que tienen en común la presencia de dificultades en la comunicación social y comportamientos restringidos y repetitivos. Sin negar la relevancia de las investigaciones que buscan conocer la etiología de este trastorno, la puesta en marcha de actuaciones para mejorar las condiciones de las personas con TEA y sus familias requiere abordar este trastorno desde una perspectiva psicosocial y cultural. Así, en el presente artículo se aborda esta condición en su triple dimensión: disease, illness y sickness. Más concretamente, y a partir de un grupo de trece familias de niños con TEA, se pretende: (1) Conocer el proceso de comunicación del diagnóstico y la información recibida (disease). (2) Evaluar la percepción de la relación con los profesionales, por parte de estas familias (illness). (3) Comprender los apoyos y obstáculos percibidos por los padres, en el abordaje del TEA (sickness). Mediante el 
empleo de preguntas abiertas y cerradas el presente estudio constata: (1) La necesidad de mejorar la comunicación del diagnóstico y mejorar la formación y sensibilización de los profesionales. (2) La importancia de empoderar a los padres como centro de atención y ofrecerles apoyos y servicios sanitarios y educativos en contextos lo más normalizados posibles. (3) Incrementar los apoyos recibidos en las diversas facetas vitales y trabajar por fomentar la aceptación social de este colectivo.

Palabras Clave: trastornos del espectro del autismo; disease; illness; sickness; familias; apoyos.

AвSTRACT: The term "autism spectrum disorders" (ASD) designates a set of varying conditions that have in common the presence of difficulties in social communication and restricted and repetitive behaviors. Without denying the relevance of research seeking to understand the etiology of this disorder, the implementation of measures to improve the conditions of people with ASD and their families needs to address this disorder from a psycho-social and cultural perspective. In this article this condition is addressed from three perspectives: disease, illness and sickness. Specifically, from a group of thirteen families of children with ASD, we aimed to: (1) Identify the process of communicating the diagnosis and providing information (disease). (2) Assess the perceived relationship with professionals of these families (illness). (3) Understand the perceived supports and barriers by parents in addressing the ASD (sickness). By using open and closed questions this study reveals: (1) The need to improve the communication of the diagnosis and the professionals' training and sensitization. (2) The importance of empowering parents as focus for care and offer support and health and education services in settings as normalized as possible. (3) Increase the support received in the various life domains and continued efforts to promote the social acceptance of this group.

KeYs words: Autism Spectrum Disorders; disease; illness; sickness; families; supports.

\section{Introducción}

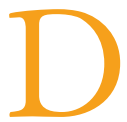

ESDE UN PUNTO DE VISTA CLÍNICO EL AUTISMO o, más propiamente dicho, los "trastornos del espectro del autismo" (TEA) designan a un conjunto de condiciones muy variables que tienen en común la presencia de dificultades en la comunicación social y comportamientos restringidos y repetitivos. Los síntomas deben estar presentes desde la infancia temprana, aunque pueden no manifestarse plenamente hasta etapas posteriores (American Psychiatric Association, 2013). En términos más aplicados Alonso Peña (2009), lo define como "una discapacidad, un trastorno generalizado del desarrollo cerebral, que produce un comportamiento anómalo, en el cual los niños afectados se muestran indiferentes, ausentes, con dificultad para formar lazos emocionales con otras personas” (p. 21). Su prevalencia parece ir en aumento en los últimos años (Autismo España, 2015; Center for Disease Control and Prevention [CDC], 2012; Fombonne, 2009). 
Cada vez son más las investigaciones que buscan conocer la etiología de este trastorno a través de estudios genéticos y neurológicos (García, Domínguez y Pereira, 2012). Sin negar la relevancia de estos estudios, de cara a la intervención, es decir, a la puesta en marcha de actuaciones para mejorar las condiciones de las personas con TEA y sus familias, también es necesario abordar este trastorno desde una perspectiva psicosocial y cultural. Como señala Baron-Cohen (2010) "Aunque el mundo empiece a adquirir conciencia de los problemas de los autistas, muchos individuos y familias siguen sin contar con un apoyo suficiente” (p. 39).

\section{Estudios interdisciplinares para la construcción social de los TEA}

Diversos estudios (p. e. Bagatell, 2010; Kaufman, 2010; Ochs et al., 2004; Ochs y Solomon, 2010; Prince, 2010) abordan el autismo desde un punto de vista interdisciplinar compartiendo dos aspectos fundamentales: (1) Poner de relieve la importancia de repensar la investigación sobre el autismo alejada de los discursos dominantes biomédicos y basada en una posición más fenomenológica y etnográfica, (2) acentuar las posibilidades de interacción social y de participación de las personas con autismo.

Las ciencias sociales se encargan de analizar las interrelaciones entre los factores o componentes biológicos, psicológicos, sociales y culturales, que configuran los procesos de enfermedad, salud y atención, utilizando un modelo bio-psico-social frente al modelo biomédico. Es necesario transformar los tradicionales enfoques biologicistas de la enfermedad, como ya plantearon autores como Kleinman (1988) y Good (1994), para incorporar la cultura a la hora de analizar y comprender el proceso salud-enfermedad-atención (Mariano, Flores y Cipriano, 2013). Este modelo bio-psico-social plantea un enfoque participativo, holístico e integral de la salud y la enfermedad, y postula que los factores biológicos, psicológicos (emociones, pensamientos y conductas) y sociales desempeñan un papel significativo de la actividad humana en el contexto de una enfermedad, trastorno o discapacidad (Engel, 1977). Dicho modelo se caracteriza por la utilización de la multidimensionalidad de la enfermedad (Comelles y Martínez, 1993) que se explica a partir de tres términos: disease, illness y sickness. Inicialmente se distinguió entre el concepto disease, considerado lo patológico, e illness, referido a la dimensión tanto cultural como social de la enfermedad (Fabrega, 1972). Posteriormente, el rechazo al etnocentrismo de la biomedicina y su abordaje como un sistema médico más, planteado por Kleinman, le permitió reformular la dualidad disease/illness de Fabrega, para darle un sentido dentro del conjunto de sistemas médicos. Así, autores, como Kleinman (1988) y Good (1994) aplicaron el término sickness a la dimensión social de la enfermedad para aludir al reconocimiento que la sociedad hace de la persona enferma y a las respuestas a su situación, por ejemplo, los procesos asistenciales que desencadena. La dimensión illness hace referencia a la dimensión cultural; la experiencia subjetiva, el sentirse enfermo y la vivencia de la enfermedad. 


\section{Disease}

La dimensión disease es relativamente sencilla de definir en el caso que nos ocupa: hablamos de TEA, una etiqueta diagnóstica para una enfermedad definida en una clasificación nosológica. Sin embargo, los problemas comienzan en el proceso de diagnóstico y comunicación de TEA, tanto para los profesionales como para los padres, partiendo además de que el diagnóstico clínico del TEA puede requerir un tiempo prolongado debido a su compleja naturaleza.

No es objeto del presente estudio hacer un repaso de las herramientas y métodos clínicos para el diagnóstico de TEA, o de los debates en torno a los cambios de los sistemas clasificatorios de esta condición en el DSM-5 frente al DSM-IV, pero sí lo es conocer la opinión de las familias acerca del proceso de diagnóstico y comunicación sobre el TEA. De la importancia de este proceso se hacen eco los trabajos de Poirier y Goupil (2008) y de Braiden et al. (2010). La atención a las preocupaciones de los padres previas a un diagnóstico es, además, fundamental para evitar las subsiguientes frustración e ira (Carbone, Behl, Azor y Murphy, 2010). En la misma línea se sitúan Osborne y Reed (2008), quienes demandan una mayor formación profesional y sensibilización acerca de los TEA, especialmente respecto a la información que los profesionales poseen y sobre sus habilidades interpersonales.

\section{Illness}

En las personas con TEA la dimensión illness alude a cómo estas personas y sus familias viven y perciben el trastorno y cómo evalúan e interpretan sus síntomas, cuáles son sus creencias, su manera de entenderlo y vivirlo, así como la forma de actuar en las diferentes situaciones sociales que se presentan en el día a día.

Al respecto Alonso Peña (2009) y Baron-Cohen (2010) relatan cómo muchos padres salen de la consulta bloqueados por la noticia. Conocer que un hijo padece TEA se convierte en una de las experiencias más duras y difíciles por las que tiene que pasar la familia. Los padres viven un tiempo en el que la esperanza de que el problema de su hijo sea pasajero coexiste con la observación del empeoramiento de los síntomas, acrecentándose el malestar familiar al no llegar a comprender lo que realmente está ocurriendo (Martínez y Cuesta, 2012). A esto se añade que al principio puede ser común en los padres la sensación de que “algo se ha hecho mal”, lo que acarrea importantes consecuencias a nivel psíquico (Rivière, 1997). Las dificultades que presentan los padres para asumir el diagnóstico van a depender de la cantidad y gravedad de síntomas que presente su hijo (Pozo, Sarria y Brioso, 2010).

Lock y Bradley (2013) señalan la importancia de incluir a la familia directamente en el tratamiento del niño. Este papel fundamental que tiene la familia desde el momento en que se diagnostica TEA en uno de sus miembros lleva a que autores como Hodgetts et al. (2013) señalen que es necesario que la atención esté centrada en todo el grupo familiar (Family-centered Care FCC), dadas las repercusiones inmediatas en las prioridades y necesidades de la familia desde el momento del diagnóstico. Es 
necesario también ampliar los estudios a la percepción que tienen los hermanos, no solo los padres. En este sentido ya pueden señalarse algunos trabajos como los de Latta et al. (2014) o Pérez y Verdugo (2008).

Algunos autores reivindican el papel de los padres como coordinadores de la atención que necesitan estos niños en los ámbitos de la educación, salud y comunidad (Carbone, Behl, Azor y Murphy, 2010). Otros autores (Hodgetss et al., 2013; Osborne y Reed, 2008) plantean la necesidad de que exista un profesional que sea capaz de coordinar esta variedad de atención que precisan. Además del personal sanitario es fundamental la labor de los profesionales educativos. En este sentido, Stoner et al. (2005) y Tucker y Schwartz (2013) mencionan barreras para facilitar la entrada de los niños, problemas en la comunicación con los equipos educativos y percepciones, en algunos casos negativas, de algunos de estos profesionales.

\section{Sickness}

Comprender la dimensión sickness en los TEA implica conocer cómo el trastorno es visto y conocido dentro de nuestra sociedad, cómo son incluidas y reconocidas las personas con autismo en la sociedad donde viven, qué servicios tanto sanitarios como educativos reciben y qué posibilidades tienen en su contexto cultural de lograr una buena calidad de vida (Grinker, 2008, 2010; Nadesan, 2005; Prince, 2010; Silverman, 2008; Sirota, 2010).

Por ejemplo, Nadesan (2005) sostiene que la idea de autismo es fundamentalmente una construcción social, que lo etiqueta como un trastorno distintivo con unas características sociales específicas. Todo ello tiene un efecto directo en las personas afectadas y en sus familias. En esta línea, Hacking (1999) concluye que el autismo es un claro ejemplo de la intersección de la biología y la cultura. Así, mientras que los factores genéticos y los aspectos neurológicos y fisiológicos no se ven afectados por su clasificación, los efectos de las expectativas de los padres, los programas de educación y las experiencias del individuo afectan y/o constituyen la expresión de los factores biogenéticos, igual que la estigmatización de la persona con autismo sigue formando parte del proceso de inventar o construir al sujeto dentro de un determinado contexto social.

La creación de asociaciones y grupos de autoayuda son fundamentales para que los padres conozcan la experiencia de otros padres que han pasado por lo mismo: por un lado, para conseguir información y eliminar preocupaciones e incertidumbres $y$, por otro, para verse reflejados en una situación de futuro donde muchos de los problemas ya han sido afrontados y superados (Martínez et al., 2010). Actualmente desempeñan un papel crucial en la lucha por los derechos e intereses de los afectados y de sus familiares y en la provisión de servicios de calidad (Díaz, 2008; Alonso Peña, 2009; Arnáiz y Zamora, 2012; Gárate, 2012).

Todos estos estudios nos hacen repensar el autismo desde la experiencia personal de los diagnosticados y sus familias, haciendo especial hincapié en las cuestiones de salud y en la política educativa. Disciplinas como la Psicología, la Antropología, la 
Terapia Ocupacional y la Educación comparten una visión del autismo como una construcción social a través de contextos ideológicos, sociohistóricos e institucionales. Por lo tanto, estos estudios demarcan un nuevo dominio interdisciplinario de investigación que examina el autismo como una visión sociocultural discutible y discutida, además de, lógicamente, como una construcción biomédica. Desde esta óptica podemos ayudar a los padres a entender mejor a sus hijos y a afrontar algunas de las preocupaciones que más les afectan sobre su futuro, relacionadas con el empleo, la integración social y la capacidad para tener una vida independiente (Carbone, Behl, Azor y Murphy, 2010). También desde esta perspectiva tienen cabida trabajos como los de King et al. (2012), quienes acentúan los efectos positivos, beneficiosos o contribuciones de tener un hijo con TEA.

Así pues y a la luz de lo expuesto previamente, consideramos que sigue siendo necesario incrementar el análisis y comprensión de los TEA como proceso cultural y social, y no sólo como proceso biomédico. Debe estudiarse bajo el paradigma multidimensional que trabaja las dimensiones disease, illness y sickness. Para ello, y a partir de la información recabada de un grupo de padres de niños afectados por TEA, esperamos:

(1) Conocer el proceso de comunicación del diagnóstico y la información recibida (disease).

(2) Evaluar la percepción de la relación con los profesionales por parte de estas familias (illness).

(3) Comprender los apoyos y obstáculos percibidos por los padres en el abordaje del TEA (sickness).

\section{Método}

\section{Diseño}

Empleando un diseño transversal, realizamos una investigación descriptiva observacional, cualitativa y cuantitativa, mediante observación participante y entrevistas realizadas en una asociación de padres de niños con TEA.

\section{Participantes}

Aceptaron participar en la investigación trece familias (de treinta y siete posibles), todas ellas pertenecientes a una asociación de niños con TEA de una provincia de Castilla y León. Respondieron 11 madres y 2 padres. 6 tenían edades comprendidas entre los 31 y 40 años, 4 entre 41 y 50 años y 3 mayores de 51 . El 54\% de los participantes tenían estudios universitarios, el 23\% Educación Secundaria Obligatoria y el otro $23 \%$ habían realizado Formación Profesional u otro estudio equivalente. La 
unidad familiar estaba formada en el $61 \%$ de las familias por cuatro miembros, en el $31 \%$ por tres y en el $8 \%$ por más de cuatro personas.

\section{Instrumentos}

Se diseñó una entrevista para los padres de 36 preguntas, de las cuales 22 son cerradas, divididas en tres bloques. Un primer bloque con preguntas sociodemográficas generales como la edad, número de miembros de la unidad familiar, estudios realizados o quién es el cuidador principal del niño. El primer bloque estaba dedicado a investigar sobre la dimensión disease y por ello planteábamos valorar el diagnóstico inicial, la información recibida y las reacciones y sentimientos frente al diagnóstico. Para analizar la dimensión illness preguntábamos sobre la relación con el hijo, dificultades y necesidades percibidas y repercusiones en la unidad familiar. En tercer lugar, para conocer la dimensión sickness, interrogábamos sobre la percepción social de los TEA, el papel de las asociaciones y grupos de autoayuda y el acceso a las ayudas sociales.

\section{Procedimiento y análisis}

El trabajo de campo con las familias se desarrolló en la asociación y centro educativo durante los seis primeros meses del año 2013. Los participantes ofrecieron su consentimiento informado tras garantizárseles el anonimato y confidencialidad de la información proporcionada. Para el estudio cuantitativo de los datos se realizaron análisis de frecuencias y descriptivos, empleando para ello el programa SPSS v. 19. Las figuras han sido elaboradas con el programa Excel v. 2007 y con el software para

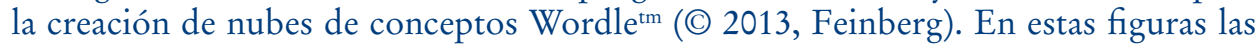
palabras que figuran en letra de mayor tamaño son las que aparecen citadas con mayor frecuencia en las entrevistas. La parte cualitativa se ha analizado mediante análisis temático por categorías elaboradas por las autoras del trabajo y asignadas por consenso.

\section{Resultados}

\section{Dimensión disease: conocimiento e información sobre el diagnóstico}

¿Cómo se enteran los padres de que sus hijos tienen problemas? Lo más habitual es que sean los propios padres quienes se den cuenta de que pasa algo. También los pediatras y los profesionales de las escuelas infantiles les avisan de la posibilidad de que su hijo tenga dificultades en el desarrollo. Lo menos habitual es que lo conozcan desde el nacimiento, en nuestro caso sólo una de las familias. Con respecto a los signos que alertaron de que el niño tenía algunas dificultades destacan: el llanto nocturno, retraso en el lenguaje, pérdida de la mirada, movimientos involuntarios e incontrolados de los ojos, estereotipias y problemas para relacionarse con otros 
niños. Como observamos en la Figura 1, las palabras en mayor tamaño de letra resaltan algunas de estas dificultades. Entre ellas "nacimiento" nos indica que a pesar del retraso en el diagnóstico clínico, los padres son conscientes desde muy pronto de que "algo no va bien".

Una de las familias comentó:

Que no se defendía con sus iguales, si le quitaban algo se lo dejaba quitar y no reaccionaba.

\section{FIGURA 1. Signos que alertaron a las familias de dificultades en el desarrollo del niño}

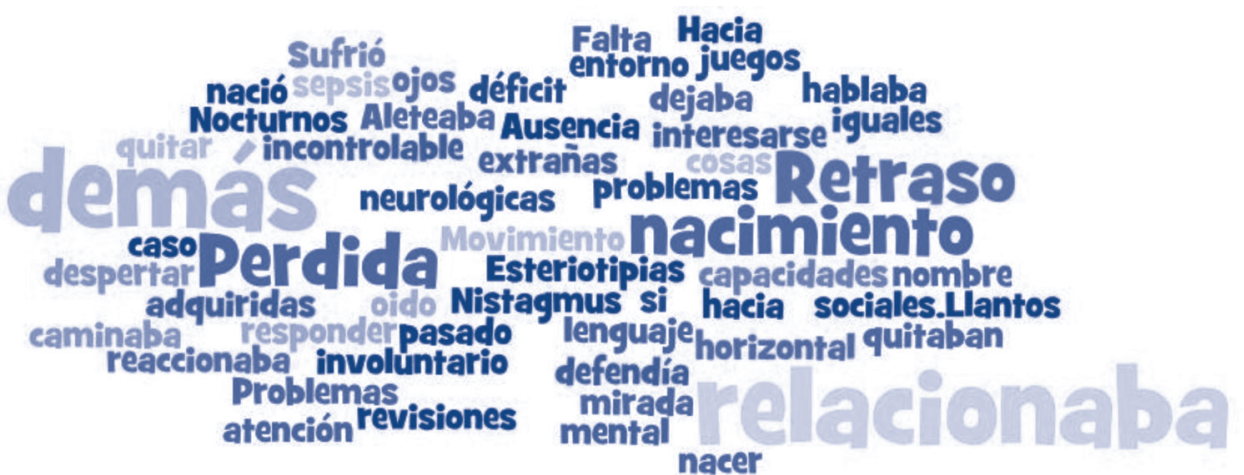

El diagnóstico inicial puede tardar en recibirse y puede variar dentro de los diferentes tipos de trastornos que incluye el TEA, en nuestro caso: trastorno autista (38\%), Trastorno Generalizado del Desarrollo no especificado (31\%), trastorno autista asociado a otros tipos de trastornos como retraso madurativo o discapacidad intelectual $(23 \%)$ y no diagnosticado de TEA, sino con otro tipo como trastorno conductual, retraso mental, retraso madurativo o síndrome de West (8\%).

Con respecto a la valoración que hacían del modo en que recibieron ese primer diagnóstico, las respuestas fueron muy diversas, encontrándose tanto respuestas positivas como negativas. Entre las primeras destacan quienes manifiestan haber recibido la información con mucho tacto, de modo muy realista, cuidadosamente, con cariño, dando ánimo:

El diagnóstico fue claro, me lo explicaron con toda la naturalidad del mundo y las perspectivas no nos las pusieron muy bien porque cada niño evoluciona de distinta manera dependiendo de las zonas del cerebro que estén afectadas.

En el polo opuesto las familias señalan que se lo comunicaron de muy mala manera, sin ninguna explicación de lo que era el trastorno y con muy poco tacto, dándoles esperanzas que luego fueron falsas o bien, poniéndoles las “cosas muy negras”: 
Nos lo dijeron como si estuviéramos en la frutería. Textual: ¿Sabes lo que es el autismo?, pues eso tiene tu hijo.

Nos dijo que nuestro hijo tenía un problema grave, muy grave y que iba a ser para toda la vida.

Nos explicaron lo que tenía, pero tampoco nos explicaron en qué consistía el trastorno. Nosotros lo único que sabíamos era que estos niños no se dejaban tocar y eso nos creó mucha confusión porque nuestro hijo era muy cariñoso y le gustaba el contacto físico.

El 69\% de las familias no desconfiaron de ese diagnóstico inicial:

El profesional que realizó el diagnóstico era de fiar y porque era evidente que a nuestro hijo le pasaba algo.

Ellos son profesionales y ven a muchos niños igual.

El 31\% que sí lo hizo señaló que se debía a que es muy duro aceptar un diagnóstico así y más después de haber recibido inadecuadas explicaciones sobre el mismo:

No se asume cuando te dan malas noticias, se piensa que los profesionales se equivocan.

Me pareció duro y no era el primer diagnóstico erróneo.

No creía que la asfixia perinatal derivara en un trastorno de carácter autista.

Más de la mitad de las familias (62\%) indicaron que no recibieron consejo, apoyo e información adicional suficiente sobre la naturaleza de los TEA al recibir el diagnóstico.

Tras haber sido informados del diagnóstico, su reacción en esta etapa inicial es muy diversa, demostrando así que el proceso de adaptación a la discapacidad es más complejo de lo propuesto por algunos autores. Sorprendentemente, la reacción más frecuentemente mencionada $(26,5 \%)$ es la de alivio por conocer lo que le pasaba a su hijo. En segundo lugar (20,6\%) destaca la reacción de depresión ["Se nos rompió el alma”], seguida de la ira y rabia ante la situación $(14,7 \%)$ ["Sensación de que me podían haber escuchado antes y no decirme que era por ser madre primeriza”]. Son también frecuentes las reacciones de incredulidad o negación o de culpabilidad (11,8\%, respectivamente), así como la de shock (8,8\%).

También son muy diversas las respuestas sobre el tipo de información que preferirían haber recibido en el momento de la comunicación del diagnóstico (Tabla 1). La mayoría piden más acceso a medios y pruebas diagnósticas, formación de los profesionales sobre el tema y orientaciones sobre cómo continuar el camino con sus hijos. Coinciden en señalar la falta de profesionales especialistas, la carencia de explicaciones sobre el desarrollo y evolución del trastorno y la ausencia de buenas instituciones que ayuden y guien a las familias:

Me hubiera gustado que nos hubieran hablado de las posibilidades, no de todo aquello que no podría hacer.

Del diagnóstico no nos importa el nombre, pero sí como orientar su vida y la nuestra, a qué centros acudir y todo ha sido por nuestra cuenta. 
Nos hubiera gustado que la primera vez que le comentamos al pediatra que había algo extraño, se hubiera puesto más interés y se le podrían haber tratado antes los ataques epilépticos.

\begin{tabular}{|l|c|c|}
\hline \multicolumn{3}{|c|}{ TABLa 1. Demandas iniciales una vez conocido el diagnóstico } \\
\hline Frecuencia & $\mathrm{N}$ & $\%$ \\
\hline Diagnóstico (pediatras) y tratamiento médico (p. e. epilepsia) precoces & 3 & 16,7 \\
\hline Falta de información o explicaciones adaptadas al receptor de la misma & 4 & 22,2 \\
\hline Falta de orientación/asesoramiento sobre trabajo a realizar con el niño & 4 & 22,2 \\
\hline Diagnóstico y atención psicológica, rehabilitadora, servicios & 3 & 16,7 \\
\hline Apoyo psicológico/orientación para padres y en transiciones (p.e. adolescencia) & 4 & 22,2 \\
\hline Total & 18 & 100,0 \\
\hline
\end{tabular}

\section{Dimensión illness: la vida después del diagnóstico}

Después de conocer el diagnóstico, la mayoría (84\%) reconocen que han sufrido un gran cambio en sus actividades cotidianas y en su vida en general. El tiempo que ha pasado desde que el niño fue diagnosticado es bastante variable, el 30\% contestaron que habían pasado menos de cinco años desde que lo recibieron, el $38 \%$ entre cinco y diez años y un $31 \%$ señalaron que más de diez años.

La madre se convierte en el cuidador principal del niño en el 61\% de los casos, solamente en un $15 \%$ son ambos padres, dependiendo del día y de la disponibilidad de cada uno. Un $8 \%$ señaló que el cuidador principal era el padre, otro $8 \%$ la madre y los hermanos y otro $8 \%$ señaló que toda la unidad familiar dependiendo de la situación del momento.

Las familias manifiestan la importancia de conocer y comprender el comportamiento del niño para poder ayudarle y adaptarse a la nueva situación. La Figura 2 representa las diferentes actitudes o comportamientos de los niños con TEA que son más difíciles de sobrellevar por parte de las familias, entre las que destacan las rabietas, las estereotipias, los chillidos, el no entender a veces lo que el niño pide o necesita y la incapacidad de poder comunicarse con su hijo:

Sus chillidos, se muerde de vez en cuando, la inactividad, no disfruta haciendo nada.

En el momento actual el que tenga tan poca movilidad, el estar sentado en silla de ruedas nos limita mucho.

El pensar que no va a ser independiente y que SIEMPRE dependes de alguien para poder dejar al niño si tienes que hacer alguna cosa extraordinaria.

El que a veces no sepa esperar, el que vayamos a los sitios y no se comporte como debe, el que le lleves la contraria y empiece a llorar... 
FIGURA 2. Comportamientos más difíciles de sobrellevar para las familias con hijos con TEA

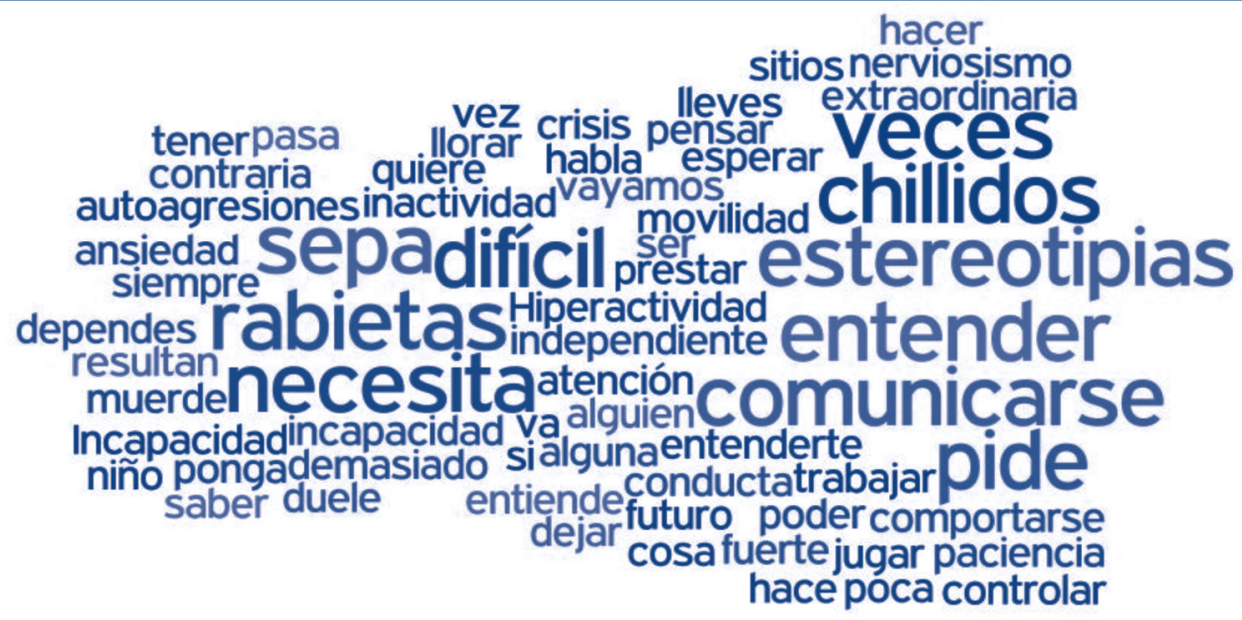

Conocer el comportamiento social del niño implica analizar su conducta en diferentes contextos, así, preguntábamos a los padres, por ejemplo, cómo se comportaban cuando acudían a las diferentes consultas médicas, y encontramos muy similar el grupo que no manifestaba conductas problemáticas (54\%) y el que sí (46\%). Influía claramente que la consulta se desarrollara en un espacio físico conocido por el niño, con personal que ya conocía y que no tuviera grandes tiempos de espera.

Dos de los aspectos más importantes que deben tenerse en cuenta a la hora de asistir a una consulta médica son la anticipación con pictogramas o dibujos y la actitud de los profesionales que les atienden, como las propias familias señalan:

Se comporta igual, pero a los médicos les pregunta si le van a poner una vacuna, si le dicen que sí se preocupa, si le dicen que no se siente aliviado, por lo demás se comporta igual.

Al dentista va una vez al año por lo que no está acostumbrado, en algunas consultas tienen mucho tacto, pero en otras no tienen conocimiento del autismo y se pone más nervioso.

A pesar de las dificultades y problemas, la mayor parte de las familias (84\%) consideran que se ha producido un desarrollo positivo de las capacidades de su hijo debido al trabajo, educación y esfuerzo constante, tanto por parte del colegio como por parte de la familia. También influyen las terapias y apoyos externos que recibe el niño, así como la rehabilitación y la medicación que toma.

$\mathrm{Al}$ apoyo que le damos y recibe por parte del centro, y al cariño que recibe que le ayuda a entender mejor las cosas. 
Sin embargo, con respecto a la medicación también nos encontramos una importante influencia negativa (24\%) debido a los efectos secundarios que provoca:

Los efectos secundarios del Risperdal hicieron que el chico diera un retroceso. En la actualidad está sentado en una silla de ruedas.

El análisis gráfico (Figura 3) de los aspectos que las familias más valoran de sus hijos nos lleva a destacar significativamente que lo que más aprecian es que son niños muy cariñosos, muy alegres cuando se encuentran bien y su gran sonrisa. Señalan que les aportan muchas cosas positivas:

Nos gusta mucho su inocencia aunque a largo plazo puede ser perjudicial para él.

Aprender a valorar lo verdaderamente importante de la vida.

Me aporta entender a más personas con cualquier discapacidad.

El entender e intentar comprender el mundo de la discapacidad.

Me ha hecho más tolerante con todo lo diferente y más paciente.

Tener a mi hija me hace ver la vida de otra manera y ser mucho mejor persona. Disfrutar de lo que realmente tiene importancia.

Apreciar las pequeñas cosas y celebrar un avance por mínimo que sea, igual que celebramos un aprobado en los exámenes de su hermana.

FIGURA 3. Actitudes de los niños más valoradas por los padres

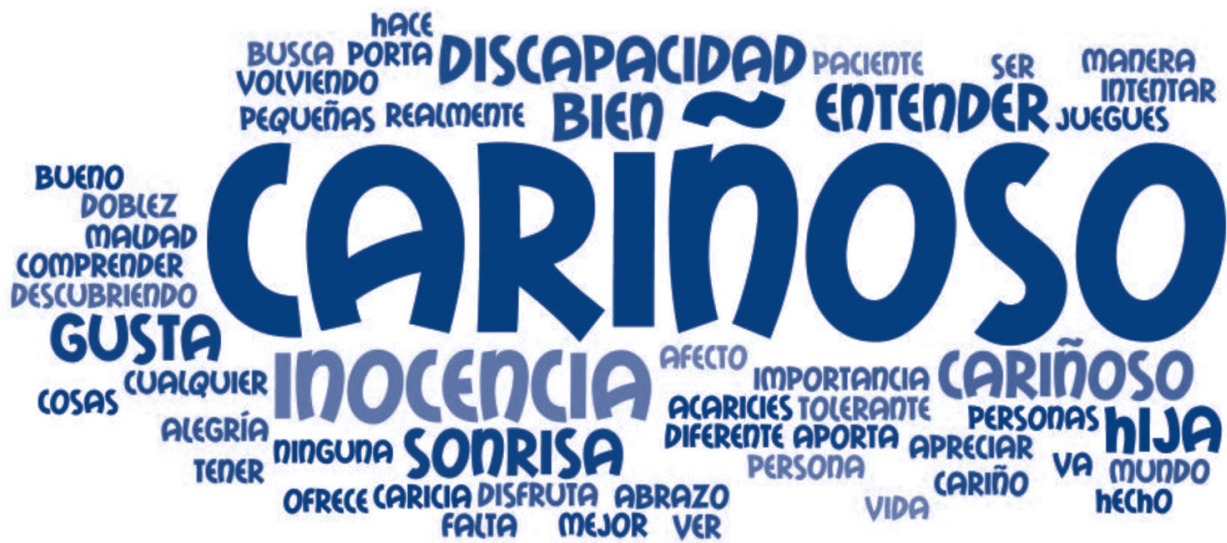

Entre las expectativas de los padres sobre el futuro de sus hijos comentaron que para que pudieran ser felices les gustaría que existiera un centro social donde sus hijos pudiesen vivir $(33,33 \%)$, que hubiera alguien que se encargara de sus hijos cuando ellos ya no estuvieran $(25,64 \%)$, que pudieran llevar una vida independiente $(23,08 \%)$ y que se encontrara un tratamiento para poder curar el trastorno $(17,95 \%)$. 
Que se le trate como a una persona con todos sus derechos.

Que le respeten en la sociedad.

\section{Dimensión sickness: respuesta de la sociedad al TEA}

El análisis de esta dimensión social nos lleva en primer lugar a conocer la atención sanitaria que reciben estos niños y sus familias. La mayor parte acude a los servicios públicos de salud, aunque un $38 \%$ también ha contratado un seguro médico privado.

Los profesionales encargados del control y seguimiento de los tratamientos son: neurólogo, neuropsiquiatra, psiquiatra y pediatra. La mayoría de las familias respondieron que habían encontrado apoyos tanto formales como informales dentro del colegio, en la familia y en conversaciones con otros padres que se encuentran en la misma situación. Más concretamente, el 54\% de las familias participantes no recibe atención psicopedagógica frente al $46 \%$ que sí la recibe. De ese $46 \%$, el 16\% de los niños llevan recibiendo esta ayuda durante un período que oscila entre dos y cuatro años; el 15\% entre cuatro y seis años, y el otro 15\% más de seis años. Sin embargo, en la mayor parte de esta ayuda psicopedagógica $(77 \%)$ las familias no realizan ninguna terapia conjunta con sus hijos. Solo el $15 \%$ acompañaban a su hijo a alguna de las sesiones de terapia psicológica o a terapias específicas, como por ejemplo la terapia asistida con perros. El porcentaje se invierte si les preguntamos acerca de las actividades de ocio y tiempo libre pues en este caso es el $85 \%$ el que comparte con sus hijos alguna actividad de este tipo, como mínimo una vez a la semana. También es relativamente frecuente que los niños acudan a logopedia.

Las familias tuvieron conocimiento de la existencia del centro de educación especial al que llevan a sus hijos por algún profesional sanitario o educacional ( $85 \%$ ) o por Internet $(15 \%)$ y valoran de forma muy positiva e imprescindible esta asistencia para el correcto desarrollo de su hijo, aunque siempre hay aspectos que pueden mejorarse:

Muy buena porque desde que está aquí tenemos más tranquilidad y estabilidad porque este es su colegio.

Positiva y muy buena. Nuestra reacción inicial era de miedo a un centro específico pues estaba escolarizado en un centro ordinario. Ahora después de cuatro años estamos contentos con la escolarización combinada.

Nuestro hijo ha mejorado bastante-dentro de sus capacidades- en el centro, así que la valoración es buena.

Todas las familias participantes reciben algún tipo de subvención o ayuda: ayuda de la ley de dependencia (54\%), beca del Ministerio para escolarización y materiales (8\%), ambas ayudas (15\%) u otro tipo de subvención, como la beca de la ONCE o una pensión de la seguridad social (23\%). Económicamente las familias alegan que el tener un hijo con TEA les supone gastos mensuales específicos importantes, entre 200 y 400 euros (46\%) o más de 400 (23\%). 
Preguntamos a los padres sobre su percepción del conocimiento que la sociedad tiene sobre los TEA y si están concienciados sobre lo que representa y mayoritariamente contestaron que no $(92 \%)$. Además piensan que no son aceptados socialmente (77\%). Solo son aceptados por su círculo familiar, y manifiestan que fuera de dicho círculo falta mucho camino por recorrer para que el conjunto de la sociedad tome conciencia de la necesidad de integración y consideración social de estos niños.

Creo que se ha avanzado respecto a épocas pasadas, pero aún se les mira con recelo en sitios públicos y ante las conductas que expresan los niños a lo largo de día.

Cada vez la gente sabe más y comprende las conductas, pero la sociedad aún no está preparada para mirar igual a todo el mundo.

Educativamente no se les integra, lo cual lleva a que socialmente no se les conozca.

Si mi hijo hace estereotipias o pega un grito, ríe o llora, parece que la sociedad se asusta, y tú escuchas de otros niños: ¡Está loco! Y personas adultas mirarte como si fuéramos bichos raros.

\section{Discusión y conclusiones}

Por lo general, son los padres quienes primero se dan cuenta de que algo les sucede a sus hijos y comienzan a buscar explicaciones. Los resultados corroboran trabajos como los de Poirier y Goupil (2008) y Braiden et al. (2010), en los que se destaca la importancia de mejorar la comunicación del diagnóstico. Así, si bien las familias entrevistadas no desconfían generalmente del diagnóstico inicial, sí reclaman la necesidad de recibir apoyo, consejo e información adicional. Plantean también la necesidad de mayor formación y sensibilización de los profesionales acerca de los TEA, en coincidencia con estudios previos (Osborne y Reed, 2010). La mayoría solicitan más información sobre acceso a medios y pruebas diagnósticas, y orientaciones sobre cómo continuar el camino con sus hijos.

A raíz del diagnóstico, la vida cotidiana de estas familias se ve claramente alterada, precisa reorganizar sus actividades diarias y asumir que el diagnóstico no es algo pasajero. Dependiendo de la cantidad y gravedad de los síntomas que presente su hijo, dicho proceso será más o menos complicado y afectará en mayor o menor medida a los miembros de la familia. Todos estos resultados coinciden con hallazgos previos (Martínez y Cuesta, 2012; Pérez y Verdugo, 2008; Pozo, Sarria y Brioso, 2010).

Martínez y Bilbao (2008) y Alonso-Peña (2009) describían que existen diferentes etapas por las que los padres pasan a la hora de recibir el diagnóstico y que hemos también constatado en nuestro trabajo, si bien las reacciones varían entre familias e incluso en una misma y ante diferentes situaciones. En cualquier caso, el apoyo, consejo y escucha por parte de los profesionales que realizan el diagnóstico es clave para asimilarlo y comenzar con estimulación temprana y terapias que ayuden al desarrollo de sus capacidades.

En coincidencia con estudios previos (Merino, García, Martínez, Olivar, Arnáiz y de la Iglesia, 2010; Osborne y Reed, 2010), otros padres ponen de manifiesto la falta 
de servicios, de profesionales especialistas, la ausencia de explicaciones sobre el desarrollo y evolución del trastorno y la inexistencia de centros e instituciones educativas especializadas.

Uno de los mayores desafíos del niño con TEA es la interacción social (Ochs et al., 2004). A menudo han sido calificados como criaturas asociales, carentes de palabras y visión del mundo (Sirota, 2010). Por ello, es necesario conocer el contexto en el que se desarrolla el niño y analizar sus comportamientos en situaciones sociales. La puesta en marcha de estrategias que les ayuden a anticipar eventos influye claramente en su conducta ante situaciones como acudir al médico o afrontar entornos poco habituales. La información proporcionada por los padres acerca de las actitudes y comportamientos más difíciles de sobrellevar, como son las rabietas, las estereotipias o las autoagresiones, plantean también prioridades para la actuación profesional de orientación y apoyo psicológico con las familias.

Los padres se consideran los coordinadores de la atención que requieren sus hijos, tanto en el ámbito de la salud, como en el educativo (Carbone, Behl, Azor y Murphy, 2010). No obstante, plantean la necesidad de que exista un profesional que se encargue de coordinar esta atención, como también se señalaba en trabajos previos (Osborne y Reed, 2008; Hodgetss et al., 2013). Como cualquier otra familia, también éstas se consideran centro de cuidados de sus hijos aunque reciban apoyos tanto formales como informales. Compartimos con Lock y Bradley (2013) la importancia de reconocer a la familia como centro fundamental de la atención que requieren estos niños y de empoderar a ésta para mejorar a su vez dicha atención. La acentuación de aspectos positivos y no sólo negativos de convivir con personas con esta discapacidad es un elemento clave para conseguir la adaptación a esta situación (King et al., 2012).

La inclusión educativa de las personas con TEA aún tiene un gran recorrido por realizar, pues esta atención se realiza fundamentalmente en centros específicos. Si bien los padres manifiestan su satisfacción con estos servicios, creemos necesario fomentar las posibilidades de que estos niños realicen actividades dentro de un centro educativo ordinario que potencien sus posibilidades de comunicación e inserción social. La integración combinada, la promoción de las aulas de apoyo, las aulas enclave y otras medidas para alumnos con necesidades de adaptación que se apartan significativamente del currículo (Peydró y Rodríguez, 2007) deben dejar espacio y garantizar su participación en actividades normales de la vida cotidiana. Debe responderse a sus necesidades en el marco más normalizado posible, entendiendo por normalización el desarrollo de un estilo de vida similar al resto de las personas de su edad y de su contexto (Ochs y Solomon, 2010).

Del presente estudio se desprende que la sociedad no tiene una adecuada percepción sobre los TEA, como corroboran mayoritariamente las familias. Continúa existiendo una clara estigmatización y se les atribuyen pautas culturales negativas, a las que tanto los afectados como sus familias tienen que hacer frente. Con todo esto planteamos, de acuerdo con Grinker (2008, 2010), que el conjunto de necesidades y problemáticas relacionadas con el TEA exige la participación e implicación del sistema no sólo sanitario, sino también social y educativo, para crear políticas comprehensivas 
de provisión de apoyos diversos y flexibles adaptados a las necesidades que presentan estas personas a lo largo de su vida.

A pesar de que el tamaño de la muestra sea reducido y no nos permita hacer generalizaciones de los resultados, el presente estudio pone de manifiesto que existen factores sociales y culturales, tal y como señalaran Hacking (1999) y Nadesan (2005), que están implicados en la identificación e interpretación de lo que significa tener TEA en nuestra sociedad.

Es cierto que no existe un tratamiento que "cure" el autismo, pero también está claro que la provisión de apoyos centrados en el niño (educativos, salud) y en su familia, así como la existencia de profesionales competentes y comprometidos y de políticas adecuadas, promoverán el éxito o “recuperación” (Broderick, 2010) de estas personas en los diferentes ámbitos vitales significativos. En consecuencia, las investigaciones al respecto deben ir más allá (Martínez, 2008) del estudio de las causas biogenéticas, para incluir también las características y relaciones culturales (illness) y sociales (sickness) asociadas a esta condición, para una mejor comprensión de los miedos, esperanzas y reivindicaciones de estas personas y de sus familiares.

\section{Referencias bibliográficas}

Alonso-Peña, J. R. (2009). Autismo y Sindrome de Asperger: Guía para familiares, amigos y profesionales (2. ${ }^{\mathrm{a}}$ ed.). Salamanca: Amarú.

American Psychiatric Association (2013). Diagnostic and Statistical Manual of Mental Disorders (DSM-5). Washington, DC: American Psychiatric Association.

Arnáiz, J. y Zamora, M. (2012). Detección y evaluación diagnóstica en TEA. En M. A. Martínez y J. L. Cuesta (Comps.), Todo sobre el autismo (pp. 89-132). Tarragona, España: Altaria.

Bagatell, N. (2010). From cure to community: Transforming notions of autism. Ethos, 38 (1), 33-55.

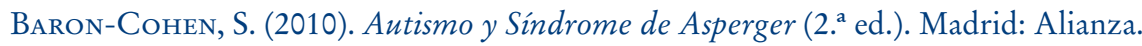

Braiden, H. J., Bothwell, J. et al. (2010). Parents' experience of the diagnostic process for autistic spectrum disorders. Child Care in Practice, 16 (4), 377-389.

Broderick, A. A. (2010). Autismo "Recuperación (a la normalidad)” y las Políticas de la Esperanza. Siglo Cero, 41 (236), 33-64.

Carbone, P. S., Behl, D. D., Azor, V. y Murphy, N. A. (2010). The medical home for children with autism spectrum disorders: Parent and pediatrician perspectives. Journal of $A u-$ tism and Developmental Disorders, 40 (3), 317-324.

Center For Disease Control (2012). Prevalence of autism spectrum disorders - Autism and developmental disabilities monitoring network, 14 sites, United States, 2008. Morbidity and Mortality Weekly Report Surveillance Summary, 61 (3), 1-19.

Comelles, J. M. y Martínez-Hernáez, A. (1993). Enfermedad, cultura y sociedad. Madrid: Eudema.

Confederación Autismo España (2015). Consultado 27 de abril de 2015. Disponible en: http://www.autismo.org.es.

Díaz, E. (2008). El asociacionismo en el ámbito de la discapacidad: Un análisis crítico. Revista Social de Pensamiento Crítico, 2 (2), 1-8.

EngeL, G. L. (1977). The need for a new medical model: a challenge for biomedicine. Science, 196 (4286), 129-136. 
Fabrega, H. (1972). Concepts of disease: logical features and social implications. Perspectives in Biology and Medicine, 15 (4), 583-616.

Fombonne, E. (2009). Epidemiology of Pervasive Developmental Disorders. Pediatric Research, 65 (6), 591-598.

GÁrATE, C. (2012). Un modelo de atención integral para las personas con TEA. En M. A. MARTínez y J. L. Cuesta (Comps.), Todo sobre el autismo (pp. 427-462). Tarragona, España: Altaria.

García, J. J., Domínguez, J. y Pereira, E. (2012). Alteraciones de la sinaptogénesis en el autismo, implicaciones etiopatogénicas y terapéuticas. Revista de Neurología, 54, 41-50.

Good, B. J. (1994). Illness representations in medical anthropology: a reading of the field. En B. J. Good, Medicine, Rationality and Experience. An Anthropological Perspective (pp. 112-122). Cambridge: Lewis Henry Morgan Lectures.

Grinker, R. R. (2008). Theme and variation: “The Discovery of the Autism”. En R. R. Grinker, Unstrange Minds. A Father Remaps the World of Autism (pp. 37-66). Cambridge: Icon Books.

Grinker, R. R. (2008). Igloos in India. En R. R. Grinker, Unstrange mind: Remapping the world of autism (pp. 197-214). Cambridge: Icon Books.

Grinker, R. R. (2010). Commentary: On being autistic, and social. Ethos, 38, 172-178.

Hacking, I. (1999). Why ask what? En I. Hacking, The Social Construction of What? (pp. 1-35). Cambridge: Harvard University Press.

Hodgetts, S., Nicholas, D., Zwaigenbaum, L. y Mcconnell, D. (2013). Parents' and professionals' perceptions of family-centered care for children with autism spectrum disorder across service sectors. Social Science y Medicine, 96, 138-146.

Kaufman, S. (2010). Regarding the rise in autism: Vaccine safety doubt, conditions of inquiry, and the shape of freedom. Ethos, 38, 8-32.

King, G., Zwaigenbaum, L., Bates A., Baxter, D. y Rosenbaum, P. (2012). Parent views of the positive contributions of elementary and high school-aged children with autism spectrum disorders and Down syndrome. Child: Care, Health and Development, 38 (6), 817-828.

Kleinman, A. M. (1988). The meaning of symptoms of disorder. En A. M. Kleinman, The Illness Narrative: Suffering, healing and the buman condition (pp. 3-46). Nueva York: Basics Books.

Latta, A., Rampton, T., Rosemann, J., Peterson, M., Mandleco, B., Dyches, T. y Roper, S. (2014). Snapshots reflecting the lives of siblings of children with autism spectrum disorders. Child: Care, Health and Development, 40 (4), 515-524.

Lock, R. H., Bradley, L., Hendricks, B. y Brown, D. B. (2013). Evaluating the success of a parent-professional autism network: Implications for family counselors. The Family Journal, 21 (3), 288-296.

Mariano, L., Flores, J. A. y Cipriano, C. (2013). Nosotros, los trastornos límite de personalidad. Narrativas y relatos de aflicción. Madrid: Círculo Rojo.

Martínez, M. A. y Bilbao, M. C. (2008). Acercamiento a la realidad de las familias de personas con autismo. Intervención Psicosocial, 17 (2), 215-230.

Martínez, M. A. y Cuesta, J. L. (2012). Todo sobre el autismo. Tarragona: Altaria.

Martínez, M. A., Cuesta, J. L., Esteban, N., Lezcano, F., Casado, R., Arnáiz, J. y Pérez De la VARGA, L. (2010). Historia y situación actual del movimiento asociativo para las personas con Trastornos del Espectro del Autismo y sus familias. Siglo Cero, 41 (4), 21-96.

Martínez-Hernáez, A. (2008). Antropología médica: teorías sobre la cultura, el poder y la enfermedad. Barcelona: Anthropos. 
Merino, M., García, M. I., Martínez, M. A., Olivar, J. S., Arnáiz, J. y De la Iglesia, M. (2010). El acceso a la prestación de asistencia sanitaria de las personas con TEA. Un problema sin resolver. Siglo Cero, 41 (236), 65-77.

Nadesan, M. H. (2005). Constructing autism: a dialectic of biology and culture, nature and mind. En M. H. Nadesan, Constructing Autism: Unravelling the 'Truth' and Understanding the Social (pp. 10-23). Nueva York: Routledge.

Ochs, E. y Solomon, O. (2010). Autistic sociality. Ethos, 38, 69-92.

Ochs, E., Kremer-Sadlik, T., Sirota, K. G. y Solomon, O. (2004). Autism and the social world: An anthropological perspective. Discourse Studies, 6 (2), 147-183.

Osborne, L. A. y Reed, P. (2008). Parents' perceptions of communication with professionals during the diagnosis of autism. Autism, 12 (3), 309-324.

Pérez, C. G. y Verdugo, M. Á. (2008). La influencia de un hermano con autismo sobre la calidad de vida familiar. Siglo Cero, 39 (227), 75-90.

Peydró, S. y Rodríguez, V. (2007). El autismo en el siglo xxi: recomendaciones educativas basadas en evidencia. Siglo Cero, 38 (222), 75-94.

Poirier, A. y Goupil, G. (2008). Perception des parents québécois sur l'annonce d'un diag nostic de trouble envahissant du développement. Journal on Developmental Disabilities, 14 (3), 29-39.

Pozo, P., Sarria, E. y Brioso, A. (2010). Contribuciones positivas que las personas con Trastorno del Espectro Autista (TEA) aportan a la familia. En M. Belinchón (Comp.), Investigaciones sobre autismo en español: Problemas y perspectivas (pp. 319-324). Córdoba: CPS-UAM.

Prince, D. E. (2010). An exceptional path: An ethnographic narrative reflecting on autistic parenthood from evolutionary, cultural, and spiritual perspectives. Ethos, 38, 56-68.

Rivière, A. (1997). Definición, etiología, educación, familia, papel psicopedagógico en el autismo. Curso de desarrollo normal y autismo. Santa Cruz de Tenerife. Disponible en: http:// www.uam.es/personal_pdi/psicologia/agonzale/Asun/2006/TrastDsllo/Lecturas/Autismo/RivDslloNorAut1.htm.

Silverman, C. (2008). Fieldwork on another planet: Social science perspectives on the autism spectrum. Biosocieties, 3, 325-341.

Sirota, K. G. (2010). Narratives of distinction: Personal life narrative as a technology of the self in the everyday lives and relational worlds of children with autism. Ethos, 38, 93-115.

Solomon, O. (2008). Language, autism, and childhood: An ethnographic perspective. Annual Review of Applied Linguistics, 28, 150-169.

Stoner, J. B., Bock, S. J., Thompson, J. R., Angeell, M. E., Heyl, B. S. y Crowley, E. P. (2005). Welcome to our world: Parent perceptions of interactions between parents of young children with ASD and education professionals. Focus on Autism and Other Developmental Disabilities, 20 (1), 39-51.

Tucker, V. y Schwartz, I. (2013). Parents' perspectives of collaboration with school professionals: Barriers and facilitators to successful partnerships in planning for students with ASD. School Mental Health, 5 (1), 3-14.

(C) Ediciones Universidad de Salamanca

Siglo Cero, vol. 46 (4), n..$^{\circ}$ 256, 2015, octubre-diciembre, pp. 7-24 